\title{
Segmentation Images Using Improved Genetic Algorithm
}

\author{
Kailasam Leelavathi ${ }^{1}$ and T. Sudha ${ }^{2}$ \\ ${ }^{1}$ Department of Computer Science, Vikrama Simhapuri University, Nellore, Andhra Pradesh, India \\ ${ }^{2}$ Department of Computer Science, Sri Padmavati Mahila Visva Vidhyalayam, Tirupati, Andhra Pradesh, India \\ E-Mail: kailasam.leelavathi@gmail.com
}

\begin{abstract}
With the expanding openness to new advancements, the principle issues in locale acknowledgment of remote detecting pictures are: (1) arrangement techniques are reliant on the division quality; and (2) the choice of delegate tests for preparing. The significant test is that the examples shown by the client are not in every case enough to characterize the best division scale. Besides, the sign of tests can be expensive, since it regularly requires visiting considered places in loco. The choice of delegate tests, then again, was bolstered in this work by the improvement of another intelligent characterization approach based on dynamic learning. Critical commitments were likewise acquired concerning the depiction of areas in remote detecting pictures by methods for: an assessment investigation of 19 descriptors; and two new methodologies for accelerating highlight extraction from a progressive system of sectioned districts.
\end{abstract}

Keywords: Segmentation Images, Hyper-spectral Imaging, Aerial Photography, Satellite Image Segmentation

\section{INTRODUCTION}

Hyper-spectral imaging is concerned with analysis and interpretation of spectra acquired from a given scene at a short, medium or long distance by an airborne or satellite sensor. This system is able to cover the wavelength region from 0.4 to 2.5 micrometers using more than two hundred spectral channels at nominal spectral resolution of 10 nanometers. Hyper-spectral Signature detects the individual absorption features of all materials, because all the materials are bound by chemical bonds. Hence hyper-spectral data is used to detect fine changes in vegetation, soil, water and mineral reflectance. Hyper-spectral remote sensing image analysis also attracts a growing interest in real-world applications such as urban planning, agriculture, forestry and monitoring.

Hyper-spectral data contain extremely rich spectral attributes, which offer the potential to discriminate more detailed classes with classification accuracy. Hyper-spectral image classification is the process used to produce thematic maps from remote sensing image. A thematic map represents the earth surface objects (Soil, vegetation, roof, road, buildings) and its construction implies the themes or categories selected for the map are distinguishable in image. Classification in remote sensing involves clustering the pixels of an image to a set of classes such that pixels in the same class are having similar properties. One of the important problems in remote sensing is huge amount of data that is typically available for processing. To combat the data explosion problem, internal and fuzzy methods were employed (Starks. S.A \& EI Paso, 2001). Majority of Image classification is based on the detection of the spectral response patterns of land cover classes.

\section{A. Problem Definition}

Remote sensing has been used in countless environmental applications with the aim of solving and improving all sorts of problems: soil quality studies, water resources research, meteorology simulations, environmental protection, among others. Remote-sensing research focusing on image classification has long attracted the attention of the remotesensing community because classification results are the basis for many environmental and socioeconomic applications.

When the landscape of a study area is complex and heterogeneous, selecting sufficient training samples becomes difficult. This problem would be complicated if medium or coarse spatial resolution data are used for classification, because a large volume of mixed pixels may occur. However, classifying remotely sensed data into a thematic map remains a challenge because many factors, such as the complexity of the landscape in a study area, selected remotely sensed data, and image-processing and classification approaches, may affect the success of a classification.

\section{RELATED WORK}

Remote sensing can be defined as collection and interpretation of information about an object, area or event without any physical contact with the object. Aircraft and satellites are the common platforms for remote sensing of earth and its natural resources (Goetz et al., 1985). Aerial photography in visible portion of the electromagnetic wavelength was the original form of remote sensing but technological developments has enabled the acquisition of information at other wavelength including near infrared, thermal infrared and microwave. Collection of information over a large numbers of wavelength bands is referred as Hyper-spectral data. Remote Sensing involves measurement of energy in various parts of the electromagnetic spectrum. A spectral band is defined as a discrete interval of the Electromagnetic spectrum. For example the wavelengths range is 0.4 micrometers to 0.5 micrometers in one spectral band. Image Classification refers to the task of extracting 
information from an image. The primary objective of image classification is to detect, identify and classify the features occurring in an image in terms of the type of class these features represent on the field [5].

Image Classification can be broadly divided into supervised and unsupervised. Many algorithms are proposed till now for image classification. It is difficult to analyze the best classification algorithm as numerous factors affect the results. The researches shows that Minimum distance classifier is highly recommended in all image classification applications (Richards 1995) due to its minimum computation time as it depends mainly on the training data, it is also said that it works best in applications where spectral classes are dispersed in feature space and have similar variance. The Fuzzy C Means clustering algorithm has been widely used in image segmentation since it was proposed [6]. In Comparison to Hard c-means algorithm FCM is able to preserve more information from the original image. However, it is noise-sensitive as it does not take into account the spatial information of a pixel [7]. The supervised Fuzzy C means as proposed for security assessments provides high accuracy and less computational effort[8].

K-Nearest Neighbor provides fast, objective, transparent and produces good results over larger areas. The importance of KNN methods is its simplicity and lack of parametric assumptions [9]. It is different from other classification methods as it does not take into account the mean of the class pixels. The literature of Maximum likelihood method describes that it needs long time of computation, relies heavily on a normal distribution of the data in each input band and tends to over-classify signatures with relatively large values in the covariance matrix [10]. However, it requires the least computational time amongst other supervised methods as the pixels that should not be unclassified become classified, and it does not consider class variability [11]. The results of supervised classification depend on the quality of training data.

In general image classification can be grouped intosupervised and unsupervised, or parametric and nonparametric,or hard and soft (fuzzy) classification, orpixel, sub pixel and per field. Supervisedclassification process is divided into two phases: (a)training phase and (b) Classification phase. In training phase, the classification algorithm is provided with information to differentiate or identify classes uniquely. This is done by assigning a limited number of pixels to the respective classes they belong to in the particular image. The file providing this information is called training data file. In classification phase, the algorithm uses the information provided in training data file by looking -for each pixel the most similar trained class and assign classes to each pixel.This behaves like a replacement for the normal classification techniques, which necessitates expensive and time-intensive field surveys [12].
Researches and studies on image classification have long been fascinated the concentration of the scientific community, from the time when many environmental and socioeconomic presentations are based on the classification consequences [13]. Usually, a classification system makes a classification map of the identifiable or meaningful features or classes of land cover sections in a part [14]. Regardless of all the advantages, classification of land-cover using multispectral imagery is a difficult subject because of the complexity of landscapes and the spatial and spectral resolution of the images being engaged. Multispectral images consist of info collected over a wide range of changes on frequencies and these frequencies change over different areas (irregular or frequency variant behavior of the signal)[15].

The overall complex nature of multispectral image data can be attributed to the spectral characteristics with correlated bands and spatial features related within the same band which is also known as the spatial correlation. An efficient method capable of arranging the spectral and spatial (contextual) info existing in the multispectral data can increase the accuracy level of the classification in a good way when matched with the traditional non-contextual information based techniques. Researches and studies on multispectral image classification have long acquired the attention of the scientific community, since most environmental and socio-economic applications are based on the classification results [16].

It is mostly likely to create groups of similar pixels found in image data into classes that match the informational categories of user interest by matching the pixels to one another and to those of the said identity [17]. Many techniques of image classification have been introduced and numerous areas like image analysis and pattern recognition use the vital term, classification. The statistical technique used for the earlier studies of landcover classification is the maximum likelihood classifier. In recent times, various studies have applied artificial intelligence techniques as seconds to the remotely-sensed image classification applications [18]. Besides, different ensemble classification technique has been presented to increase the classification precision [19].

\section{PROPOSED SYSTEM}

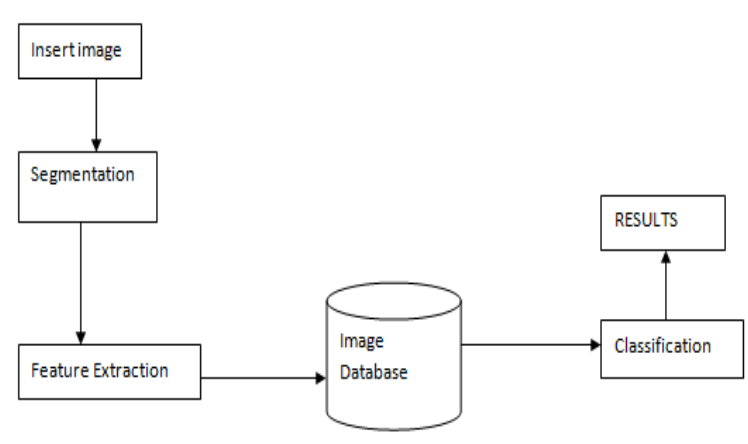

Fig. 1 Proposed System interface 
A. Algorithm: Algorithm Improved Genetic classification algorithm

$\mathrm{T}{ }^{\cdots} 0$

Select initial training Set

Evaluate database $\mathrm{D}(\mathrm{T})$

while not 'termination-condition' do

$\mathrm{T} \cdots \mathrm{T}+1$

if 'add_conditions_criterion' then

add condition to images in database

end if

Select sub database from $\mathrm{D}(\mathrm{T}-1)$ : $\mathrm{D}(\mathrm{T})$

Apply recombination operators on

individuals of $\mathrm{D}(\mathrm{T})$

Evaluate $\mathrm{P}(\mathrm{T})$

if 'found_new_best_tree' then

store copy of new best tree

end if

end while

end algorithm

\section{FEATURE EXTRACTION}

Feature extraction process for satellite image segmentation is discussed in this section. Four new segments like as road, building, tree and shadow are obtained from the 36 segments. Locale developing division is a way to deal with look at the neighboring pixels of the underlying "seed focuses" and decide whether the pixels are added to the seed point or not. [20]

Step1. Choosing an arrangement of at least one beginning stage (seed) frequently can be founded on the idea of the issue.

Step2. The district is developed from these seed focuses to adjoining point contingent upon a limit or criteria(8associated) we make.

Step3. District development should stop when no more pixels fulfill the criteria for incorporation in that locale.

At that point we can close a few critical issues about locale growing

1. The appropriate choice of seed focuses is imperative. The choice of seed focuses is relying upon the clients.

2. More data of the picture is better. Clearly, the network or pixel nearby data is useful for us to decide the limit and seed focuses.

3. The esteem, "least territory limit". No district in locale developing technique result will be littler than this limit in the fragmented picture.

4. The esteem, "Closeness limit esteem". On the off chance that the distinction of pixel-esteem or the distinction estimation of normal dim level of an arrangement of pixels not as much as "Likeness limit esteem", the districts will be considered as an equivalent area.

5. The aftereffect of a picture after district developing still have point's dark level higher than the edge however not associated with the question in picture.

\section{RESULTS}

The following results shows that the clear difference between the original image and segmented image. The results are in the image 1(a) and 1(b). The below Table I shows that the Accuracy Percentage of the proposed system and existing system.

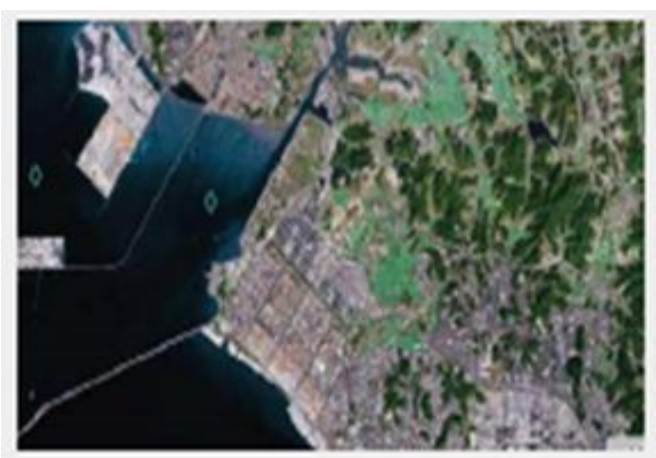

Fig. 1 a) Original Image

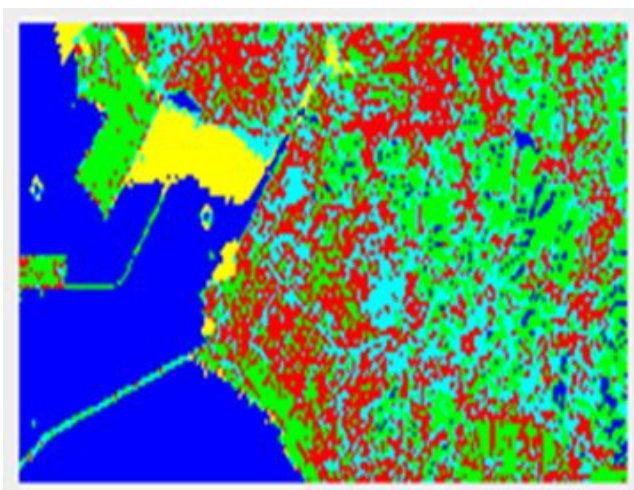

Fig. 1b) Segmented Image

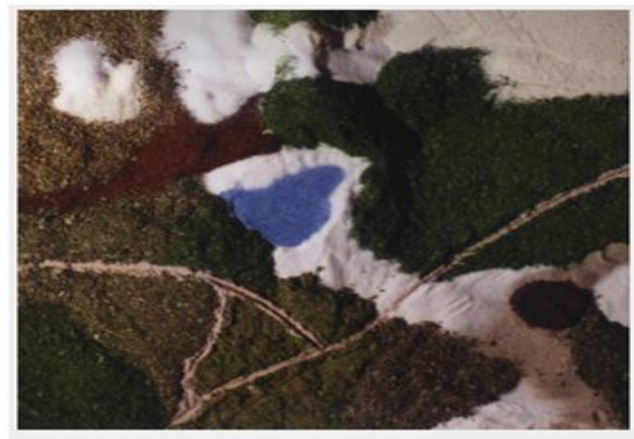

Fig. 2 a) Original Image

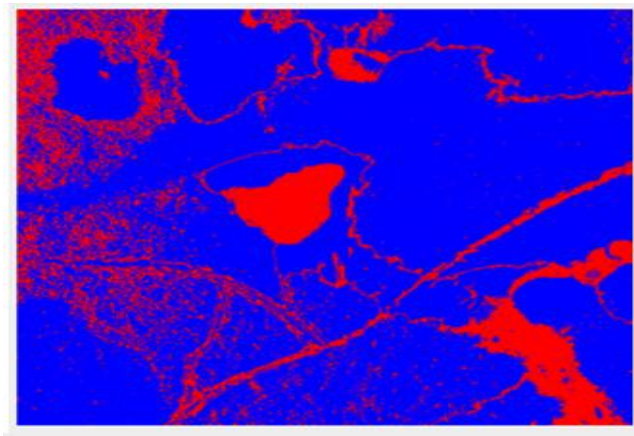

Fig. 2b) Segmented Image 
TABle I EXISTING AND PROPOSED System ACCURACIES IN PERCENTAgeS

\begin{tabular}{|c|l|c|}
\hline Set & Existing & proposed \\
\hline 1 & 94.4 & 89.9 \\
\hline 2 & 93.1 & 88.2 \\
\hline 3 & 90.3 & 87.3 \\
\hline 4 & 93.5 & 89.3 \\
\hline 5 & 94.1 & 90.3 \\
\hline 6 & 93.8 & 90.7 \\
\hline
\end{tabular}

\section{CONCLUSION}

In this study we proposed that a novel district shrewd profound component portrayal system for remote detecting pictures. In this approach, the objective related bouncing boxes are first registered for the applicant areas and a profound CNN display is connected to extricate the territorial profound highlights for each picture. At that point, the territorial profound highlights are encoded into a solitary element vector for each picture by an enhanced calculation, where a weighted multi-neighbor task technique is proposed to ascertain the portrayal. The primary focal points of the our proposed approach are: (1) speaking to the pictures with district savvy profound highlights can catch the neighborhood geometric invariance of target data all the more precisely and hold more particular substance data in the last picture highlights. (2) The enhanced calculation takes the area score and visual word task weight into thought when encoding the nearby local highlights and in this way can produce more viable extraordinary element vectors for conclusive picture portrayals. Broad analyses on two distinctive remote detecting picture investigation assignments have shown the prevalence of our methodology over the conventional element speaking to techniques.

\section{REFERENCES}

[1] K.Perumal and R.Bhaskaran, "SVM-Based Effective Land Use Classification System For Multispectral Remote Sensing Images", (IJCSIS)International Journal of Computer Science and Information Security, Vol. 6, No. 2, pp.95-107, 2009.

[2] Jan Knorn, Andreas Rabe, Volker C. Radeloff, Tobias Kuemmerle, JacekKozak, and Patrick Hostert, "Land cover mapping of large areas using chain classification of neighboring Landsat satellite images", Remote Sensing of Environment, Vol. 118, pp. 957-964, 2009.

[3] XiaochenZou, and Daoliang Li, "Application of Image Texture Analysis to Improve Land Cover Classification", WSEAS Transactions on Computers, Vol. 8, No. 3, pp. 449-458, March 2009.

[4] Reda A. El-Khoribi, "Support Vector Machine Training of HMT Models for Multispectral Image Classification", IJCSNS International Journal of Computer Science and Network Security, Vol.8, No.9, pp.224-228, September 2008.

[5] B.Sowmya and B.Sheelarani, "Land cover classification using reformed fuzzy C-means", Sadhana, Vol. 36, No. 2, pp. 153-165, 2011.

[6] V.K.Panchal, Parminder Singh, NavdeepKaur and Harish Kundra, "Biogeography based Satellite Image Classification", International Journal of Computer Science and Information Security IJCSIS, Vol. 6, No. 2, pp. 269-274, November 2009.
[7] Huang B, Xie C, Tay R, and Wu B, "Land-use-change modeling using unbalanced support-vector machines", Environment and Planning B: Planning and Design, Vol. 36, No. 3, pp.398-416,2009.

[8] James A. Shine and Daniel B. Carr, "A Comparison of Classification Methods for Large Imagery Data Sets", JSM 2002 Statistics in an ERA of Technological Change-Statistical computing section, New York City, pp.3205-3207, 11-15 Aug. 2002.

[9] D. Lu, and Q. Weng, "A survey of image classification methods and techniques for improving classification performance", International Journal of Remote Sensing, Vol. 28, No. 5, pp. 823-870, January 2007.

[10] M. Govender, K. Chetty, V. Naiken and H. Bulcock, "A comparison of satellite Hyper-spectral and multispectral remote sensing imagery for improved classification and mapping of vegetation", Water SA, Vol. 34, No. 2, April 2008.

[11] M.F.Jasinski, "Estimation of subpixel vegetation density of natural regions using satellite multispectral imagery", IEEE Transactions on Geoscience and Remote Sensing, Vol. 34, pp. 804-813, 1996.

[12] C. Palaniswami, A.K. Upadhyay and H.P. Maheswarappa, "Spectral mixture analysis for sub-pixel classification of coconut", Current Science, Vol. 91, No. 12, pp. 1706 -1711, 25 December 2006.

[13] S. Chen, S.R. Gunn, and C.J. Harris,' The Relevance Vector Machine Technique for Channel Equalization Application', IEEE transactions on neural networks, Vol. 12, No. 6, November 2001.

[14] Pak-Kin Wong, QingsongXu,' Rate-Dependent Hysteresis Modeling and Control of a Piezostage Using Online Support Vector Machine and Relevance Vector Machine', IEEE transactions on industrial electronics, Vol. 59, No. 4, April 2012.

[15] BehnoodGholami, "Relevance Vector Machine Learning for Neonate Pain Intensity Assessment Using Digital Imaging", IEEE transactions on biomedical engineering, Vol. 57, No. 6, June 2010.

[16] Mahesh Pal and Giles M. Foody,' Evaluation of SVM, RVM and SMLR for Accurate Image Classification with Limited Ground Data', IEEE journal of selected topics in applied earth observations and remote sensing, Vol. 5, No. 5, October 2012.

[17] Andreas Ch. Braun, Uwe Weidner, and Stefan Hinz,' Classification in High-Dimensional Feature Spaces-Assessment Using SVM, IVM and RVM with Focus on Simulated EnMAP Data', IEEE journal of selected topics in applied earth observations and remote sensing, Vol. 5, No. 2, April 2012.

[18] F.A. Mianji, Ye Zhang, and A.Babakhani, 'Nonlinear discriminant analysis and RVM for efficient classification of small land-cover patches',IEEE Conference Publications Communications and Signal Processing (ICCSP), 2011.

[19] Uehara, Hisashi; Watanabe, Hideyuki; Katagiri, Shigeru; and Ohsaki, Miho, 'Comparison between Minimum Classification Error training and Relevance Vector Machine,IEEE Conference Publications TENCON 2012 - 2012.

[20] A. Babaeean, A.B. Tashk, M.Bandarabadi, S. Rastegar, 'Target Tracking Using Wavelet Features and RVM Classifier', IEEE Conference Publications Natural Computation, 2008. ICNC '08.

[21] G. Camps-Valls and L. Bruzzone, "Kernel-based methods for Hyperspectral image classification,"IEEE Trans. Geosci. Remote Sens. Vol. 43, No. 6, pp. 1352-1362, Jun. 2005.

[22] M.E. Tipping, "The relevance vector machine,"in Advances in Neural Information ProcessingSystems, Vol. 12, S.A. Solla, T.K. Leen, and K.R. Müller, Eds. Cambridge, MA: MIT Press, 2000.

[23] Ming-Hseng Tseng, Sheng-Jhe Chen, Gwo- Haur Hwang, and Ming$\mathrm{Yu}$ Shen, "A genetic algorithm rulebased approach for land-cover classification", Journal of Photogrammetry and Remote Sensing ,Vol.63, No. 2, pp. 202-212, 2008.

[24] Pall Oskar Gislason, Jon AtliBenediktsson, and Johannes R. Sveinsson, "Random Forests for land cover classification", Pattern Recognition Letters, Vol.27, No. 4, pp. 294-300, 2006.

[25] K.Leelavathi, and T.Sudha"Remote image classification using improved deccission tree and neural networks",IJECIERD, Vol.5, April 2015.

[26] K.Leelavathi, and T. Sudha, "Improving accuracy in Spatial images classification IJCSEITR, Vol.5, pp. 43-48, June 2015. 